\title{
THE PRODUCTION OF $\mathrm{H}_{2} \mathrm{~S}-\mathrm{NEGATIVE} \mathrm{STRAINS} \mathrm{OF} \mathrm{SALMONELLA}$
}

\author{
By W. JAMES WILSON, Queen's University and Water Office Laboratories, Belfast
}

The production of $\mathrm{H}_{2} \mathrm{~S}$ is one of the characteristics of most members of the Salmonella group. Such members were shown by me (1923) to reduce sodium sulphite to sulphide in the presence of glucose and iron salts. In the same paper, attempts were made to devise a medium based on this finding by which reducing organisms, e.g. S. typhi could be isolated from faeces, but the ascertainment that certain strains of Bact. coli were also reducers made the problem more difficult. This difficulty was surmounted by my discovery (Wilson \& Blair, 1927) that salts of bismuth in the presence of sulphite had an inhibitory action on the growth of most of the Bact. coli reducing strains and of anaerobes, e.g. C. welchii, and led to the development of the bismuth-sulphite medium of Wilson \& Blair (1927, 1931).

The Wilson \& Blair medium and its modifications have proved their value in all parts of the world in the isolation of $S$. typhi, S. paratyphi B and the great majority of food-poisoning bacteria belonging to the Salmonella group; the truth of the latter statement is shown by the recent (1947) Report of the Medical Research Council on the bacteriology of spray dried egg, with particular reference to food poisoning. In 1923 I had already found that exceptions existed, in that $B$. suipestifer Uhlenhuth (salmonella, type Hirschfeld), B. suipestifer (salmonella type G), $B$. abortio-equinuus ' 626 Foal', $B$. voldagsen Wegener (salmonella, type Hirschfeld), $B$. gallinarum, B. pullorum, B. sanguinarium and $B$. paratyphosus A were non-reducers.

As regards $S$. typh $i$ thousands of strains have been isolated on the medium in all parts of the world and only a few investigators have referred to the finding of non-reducing $\mathrm{H}_{2} \mathrm{~S}$-negative strains. I have met with strains that did not give as dark colonies as usual but found on further study that they had not completely lost their capacity for $\mathrm{H}_{2} \mathrm{~S}$ production. In the present paper I shall describe the production of $\mathrm{H}_{2} \mathrm{~S}$-negative variants of $S$. typhi and $S$. paratyphi $\mathrm{B}$ in sulphite-bismuth broth, but before doing so I shall refer to a study of two atypical strains of S. typhi by Sage \& Spaulding (1942); these two strains on isolation fermented lactose and sucrose but $\mathrm{H}_{2} \mathrm{~S}$ was not produced. Only after 1 year of artificial cultivation did they become entirely typical. Periodic faeces isolation from one case, a carrier, revealed a gradual transition from the abnormal biochemical pattern first observed to a completely typical one 3 years later. Serologically both strains possessed considerable ' $\mathrm{Vi}$ ' antigen but when isolated only a small amount of flagellar antigen. No other unusual antigenic property was observed. Sera from patient J.O. showed a gradual increase in somatic and flagellar typhoid antibodies, whereas the second patient (T.R.) during her entire hospital stay failed to develop a titre above 1:20 against S. typhi TO901 antigen. Both patients presented the usual symptoms at onset and subsequently pursued the customary course of typhoid fever.

In the recent works of Dubos (1946), Luria (1947) and Braun (1947) in which bacterial variation and bacterial dissociation are reviewed $I$ can find no reference dealing with $\mathrm{H}_{2} \mathrm{~S}$-variation of $S$. typhi, except that of Sage \& Spaulding outlined above, and an account given by Vietorisz (1937) of different types of colonies of $S$. typhi on the Wilson-Blair medium, the type according to the writer being influenced by cystine in an enrichment medium.

Saphra \& Seligmann (1947) from a case of fatal meningitis in a baby 6 months old isolated a coliform lactose fermenting bacillus with complete antigen of $S$. newington which was at first $\mathrm{H}_{2} \mathrm{~S}$ negative but on further culture became $\mathrm{H}_{2} \mathrm{~S}$-positive.

It was while experimenting with sulphite-bismuth broth enrichment media for the culture of TO 901 strain of $S$. typhi and the subculture of the resulting organisms that I observed the appearance of discrete clear colonies instead of the usual black colour on the bismuth-sulphite medium.

The solid bismuth-sulphite medium employed for subculture from the bismuth-sulphite broth was a slight modification of that recommended by me in 1938. A stock bismuth-sulphite solution is prepared by dissolving $30 \mathrm{~g}$. bismuth-ammonio-citrate scales in 250 c.c. boiling distilled water and mixing this with a solution obtained by boiling $100 \mathrm{~g}$. sodium sulphite anhydrous in 500 c.c. of water and then while the mixture is boiling there is added $100 \mathrm{~g}$. of sodium-phosphate crystals $\mathrm{Na}_{2} \mathrm{HPO}_{4} \cdot 12 \mathrm{H}_{2} \mathrm{O}$. To this mixture when cool there is added a solution of glucose, obtained by dissolving $50 \mathrm{~g}$. of commercial glucose in the 250 c.c. of boiling distilled water. To prepare the medium add to 100 c.c. of hot melted $2 \%$ nutrient agar 20 c.c. of stock mixture, then 4.5 c.c. of $1 \%$ solution of iron citrate to every 200 c.c. of which there have been added 25 c.c. of a $1 \%$ solution of brilliant green in distilled water 
and pour into Petri dishes. On the surface of the medium (W.B.) $S$. typhi and S. paratyphi B form black colonies often surrounded by a dark halo.

I have found that the colonies especially of S. paratyphi and of Salmonella food poisoning organisms grow more quickly (within $18 \mathrm{hr}$.) and that the black halo is more pronounced when 4 c.c. of a laked blood solution is added to 100 c.c. of the melted agar cooled to $60^{\circ} \mathrm{C}$. The blood mixture, which keeps well, is prepared by adding to 700 c.c. of sheep's blood containing $1 \%$ sodium citrate 300 c.c. of water in which 10 g. of sodium bisulphite has been dissolved and finally 30 c.c. of ether. This bisulphite blood mixture is also useful in the preparation of blood tellurite plates for the culture of $B$. diphtheriae.

The addition of the blood removes to some extent the inhibition of the growth of enterococci and some strains of Bact. coli, but the more rapid growth of Salmonella group and the greater darkening of the colonies is ample compensation. The addition of a larger amount of brilliant green is tolerated in the presence of the blood, so that 1 c.c. of a $1 \%$ brilliant green can be added besides that contained in the iron citrate brilliant green solution. This procedure suppresses the growth of most of the enterococci and Bact. coli. For convenience this modification of the W.B. medium is referred to in this paper as the B.W.B.

\section{EXPERIMENTS}

In the first two experiments to 100 c.c. of nutrient broth there were added $1 \cdot 2 \mathrm{~g}$. sodium sulphite anhydrous, $0.025 \mathrm{~g}$. bismuth-ammonio-citrate scales and $0.5 \mathrm{~g}$. glucose. In the last few experiments mannite replaced glucose and in some there were added to every 100 c.c. four drops of a $4 \%$ iron citrate solution. A mannite powder which had been prepared on 2. iii. 45 was found satisfactory; it consisted of $24 \mathrm{~g}$. sodium sulphite anhydrous, $0.5 \mathrm{~g}$. bismuth-ammonio-citrate scales, and $10 \mathrm{~g}$. mannite. To 100 c.c. of broth 1.7 g. of this powder was added and the mixture boiled and divided into tubes each containing 10 c.c. The tubes were heavily inoculated from culture on agar, incubated at $38^{\circ} \mathrm{C}$. and from time to time subcultures were made on the B.W.B. medium. If clear colonies developed they were subcultured on MacConkey's medium, and then tested as regards their reducing power by stab inoculation in my (1923) glucose iron sulphite agar medium consisting of 100 c.c. nutrient agar to which had been freshly added 15 c.c. of a glucose sulphite solution and l c.c. of an $8 \%$ watery solution of ferrous sulphate crystals. The glucose sulphite solution which keeps for months is prepared by boiling $40 \mathrm{~g}$. sodium sulphite anhydrous in 200 c.c. of distilled water and mixing it when cool with 100 c.c. of distilled water in which $20 \mathrm{~g}$. of glucose had been dissolved. Sulphite reducing Salmonella show in stab cultures after a few hours in the incubator, a black line.

\section{Experiment 1}

On 12. iv. 47 sulphite-glucose-bismuth broth was inoculated with $S$. typhi TO 901. On 14. iv. 47 subcultures on B.W.B. showed only black colonies: on 15. iv. 47 and 25. iv. 47 many of the colonies were clear and free from any dark halo. On 29. iv. 47 no growth developed when subculturing was attempted. The growth was never profuse and the medium in the absence of iron was slowly bactericidal.

\section{Experiment 2}

This was a repetition of Exp. 1 with the same result. Tubes were inoculated on 18. iv. 47. Subcultures on 22. iv. 47 yielded non-reducing colonies the bacteria of which were non-lactose fermenters and which were agglutinated by a typhoid agglutinating serum. On 25 . iv. 47 the culture was dead.

\section{Experiment 3}

On 26. iv. 47 broth tubes containing mannitebismuth-sulphite powder and iron were inoculated with S. typhi (TO901) and with laboratory stock strain of $S$. paratyphi B. On 28. iv. 47, 30. iv. 47 and on 6.v. 47 among the black colonies developing on subculture there were pale non-reducers both in the typhoid and paratyphoid plates. These non: reducing organisms were agglutinated to the same titre by typhoid and paratyphoid B.O. sera as were the original reducing strains.

\section{Experiment 4}

Tubes similar to those employed in Exp. 3 but without addition of iron were inoculated with S. typhi TO 901, with four strains of recently isolated typhoid cultures and with four strains of recently isolated paratyphoid cultures. Subcultures were made at intervals of days but no clear non-reducing colonies appeared in the case of the recently isolated cultures, but in the case of S. typhi TO 901 which at first showed only black colonies, all the colonies on 10. vi. 47 were clear and consisted of non-reducers.

\section{Experiment 5}

Broth containing mannite-sulphite-bismuth powder and four drops of an iron solution was tubed, and tubes inoculated on 2. vii. 47 with (1) S. typhi TO901, (2) stock S. paratyphi B, (3) a recently isolated typhoid bacillus (Campbell), (4) a recently isolated S. paratyphi B (Parks), (5) a recently isolated S. paratyphi B (Hill). Subcultures on 8. vii. 47 showed no non-reducers except those of S. typhi TO 901 and S. paratyphi B (Parks). On 
11. vii. 47 subcultures on B.W.B. showed that S. typhi TO901 and S. paratyphi B (Parks) consisted entirely of non-reducers and that a few nonreducers were present in the $S$. typhi (Campbell) tube. On 22. vii. 47 subcultures showed that $S$. typhi TO 901 and S. typhi (Campbell) were dead, that the S. paratyphi $\mathrm{B}$ (Parks) contained living non-reducers and that stock $S$. paratyphi $\mathrm{B}$ and $S$. paratyphi $\mathrm{B}$ (Hill) showed only reducers.

\section{Experiment 6}

On 11. viii. 4750 c.c. of broth in which $0.9 \mathrm{~g}$. sulphite-bismuth-mannite powder had been boiled and to which four drops of a $4 \%$ solution of ferri citrate scales had been added were divided into tubes which were inoculated with cultures from agar plates of S. typhi TO 901 and stock S. paratyphi B. The tubes were 6 for $S$. typhi and 6 for S. paratyphi B. The agar plates had been kept at room temperature for varying lengths of time. It was thought that the age of the inoculum might have some influence on the production of the variants and the conclusion was reached that perhaps in the case of $S$. typhi 901 this was the case; in the case of $S$. paratyphi B no variants were detected in this experiment. No. 1 tubes were inoculated with growth from plates of 11. iv. 47, similarly nos. $2,3,4,5$ and 6 tubes from plates of 28. v. 47,18 . vi. 47,9 . vii. 47 and 4 . viii. 47 respectively. Subcultures on the B.W.B. from the S. paratyphi B tubes from 14. viii. 47 to 30 . viii. 47 when the experiment ended all yielded black and no clear non-reducing eolonies. In the case of the S. typhi TO 901 tubes subcultures from no. 1 tube yielded amongst numerous black, 2 clear non-reducing colonies on 14. viii. 47 whereas all the colonies from tubes 2, 3, 4, 5 and 6 were black. (See table below.)

On 26. xi. 47 the cultures employed and the strains developing in the course of the experiments were re-examined. No changes in the fermentation

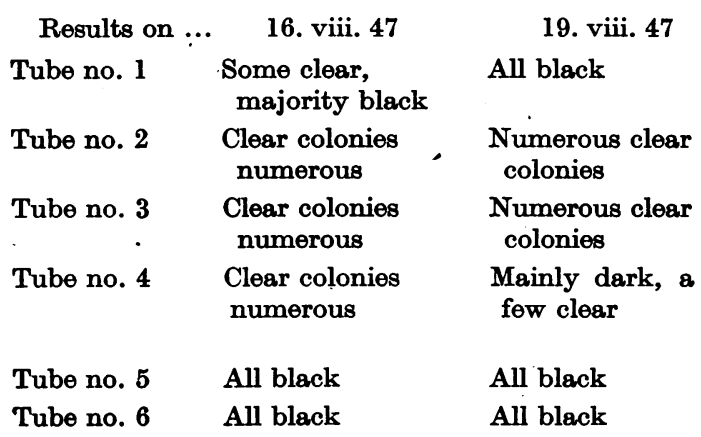

reactions were observed; as regards the growth on agar that of the non-reducing S. typhi TO901 was more luxuriant than that of the parent reducer: the non-reducer $S$. paratyphi B was more motile than the original culture. Serologically there was no marked difference between the original cultures and their variants. S. typhi TO 901 and S. paratyphi B and their variants showed the same titre, but the non-reducer $S$. paratyphi B was more rapidly agglutinated than its parent and evidently possessed much flagellar antigen: the agglutination titre of S. typhi non-reducer Campbell was half of that of the original reducing strain.

It would appear that certain strains of Salmonella give rise to variants or mutants and that these can be readily detected in a broth culture in which a sulphite is reduced to a sulphide. It is probable that the non-reducing variants have a better chance of survival in the altered medium which eventually is liable to cause their destruction. The demonstration of variants is not always successful and it is probable that the formation does not always occur.

More investigations would be necessary to ascertain the optimum medium for their occurrence, and also to discover whether their pathogenicity was affected in any way.

Their occurrence under natural conditions is so rare that the value of the bismuth-sulphite medium of Wilson \& Blair is not seriously depreciated.

\section{SUMMARY}

It has been shown that certain strains of Salmonella grown in nutrient broth containing sulphite and bismuth, develop variants that are incapable of reducing sulphite to sulphides in the media of Wilson \& Blair.

The altered strains showed no alterations in their antigenic and biochemical characters.

\begin{tabular}{|c|c|c|}
\hline 23. viii. 47 & 27. viii. 47 & 30 viii. 47 \\
\hline $\begin{array}{l}\text { Majority black, a } \\
\text { few clear }\end{array}$ & $\begin{array}{l}\text { One clear, rest } \\
\text { black }\end{array}$ & $\mathrm{Nil}$ \\
\hline Majority clear & 1 black, 17 clear & Nil \\
\hline $\begin{array}{l}\text { Hundreds of clear, } \\
10 \text { black }\end{array}$ & 1 black, 20 clear & $\mathrm{Nil}$ \\
\hline $\begin{array}{l}\text { Hundreds of } \\
\text { colonies, } 2 / 3 \text { clear, } \\
1 / 3 \text { black }\end{array}$ & 3 black, 17 clear & Nil \\
\hline All black & All black & Nil \\
\hline All black & All black & All black \\
\hline
\end{tabular}




\section{REFERENCES}

Braun, W. (1947). Bacterial dissociation. Bacteriological Reviews, II, 2, 1.

Dubos, Rente J. (1946). The Bacterial Cell. Cambridge, Mass. : Harvard University Press.

LURIA, S. E. (1947). Recent advances in bacterial genetics. Bacteriological Review, II, 1, 1.

Medical Research CouncIl (1947). The bacteriology of spray-dried egg with particular reference to food poisoning. Spec. Rep. Ser., no. 260. London: H.M. Stationery Office.
Sage, D. N. \& Spaulding, E. H. (1942). J. Bact. 44, 647.

SaPHra, I. \& SeligmanN, E. (1947). J. Bact. 54, 270.

Vietorisz, K. (1937). Zbl. Bakt. (1.Abt. Orig.), 139, 151.

Wruson, W. J. (1923). J. Hyg., Camb., 21, 392.

Wrison, W. J. (1938). J. Hyg., Camb., 38, 507.

Wilson, W. J. \& BlaIr, E. M. McV. (1927). J. Hyg., Camb., 26, 374.

Wruson, W. J. \& BlarR, E. M. McV. (1931). J. Hyg., Camb., 31, 139 .

(MS. received for publication 10. xu. 47.-Ed.) 